\title{
Design of A Multifunctional and Cost-Effective Wideband Planar Antenna Array System for Multiple Wireless Applications
}

\author{
Ming-Iu Lai ${ }^{(1)}$, Chin-Feng Liu ${ }^{(1)}$ and Shyh-Kang Jeng ${ }^{(2)}$ \\ (1) Graduate Institute of Communication Engineering, \\ National Taiwan University, Taiwan, R.O.C. \\ milai1102@yahoo.com.tw \\ (2) Graduate Institute of Communication Engineering and \\ Department of Electronic Engineering, \\ National Taiwan University, Taiwan, R.O.C. \\ skjeng@ew.ee.ntu.edu.tw
}

In this paper, a new wideband array antenna system for multiple wireless applications is proposed. It is composed of two equispaced four-element arrays, phase shifters, diplexers, and power dividers. The two arrays are interleaved and connected to diplexers and power dividers. The proposed antenna system associated with feeding network and antenna elements can be integrated in a single substrate. Consequently, such a design is with low fabrication and assembly cost and compact size. A wideband antenna array with bandwidth from $2.0 \mathrm{GHz}$ to $6.0 \mathrm{GHz}$ and main beam pointing to 20 degrees is illustrated as an example.

\section{Introduction}

Recently wireless communication products emerge in large number and gradually become living essentials. The demand of various base stations and access points for different communication standards increases in order to pursue better service quality. As the number of base stations and access points goes up, the number of antennas increases dramatically. Technically, it results in serious couplings between antennas of different communication systems and occupies a large space. For the public, it arouses concerns about their excessive electromagnetic radiation. Thus, a compact multifunctional array antenna to provide a wide variety of wireless services is highly desired.

Consider an equispaced array of 4 elements, the array factor can be written in the form [1]

$$
\begin{gathered}
A F(f, \theta)=\sum_{n=1}^{4} e^{j n\left(k_{0} d \sin \theta-\delta\right)} \\
\theta_{0}=\sin ^{-1}\left(\frac{\delta}{k_{0} d}\right)
\end{gathered}
$$

In the above equations, a uniform progressive phase factor $\delta$ is introduced to position the main beam to angles $\theta_{0}$ and $\pi-\theta_{0}$. If $\delta$ is a linear function of frequency, the direction of main beam still points at $\theta_{0}$ and $\pi-\theta_{0}$, but the shape 
of main beam deforms and the level of side lobe degrades as frequency sweeps. The digital signal processing technique, digital beamforming, can provide a frequency-invariant beam pattern over an arbitrary bandwidth [2]. This approach is very powerful, but for an n-element array each antenna requires an $\mathrm{RF}$ transceiver and $\mathrm{A} / \mathrm{D}$ converter, resulting in high fabrication cost. Consequently, designing an array with lower cost and beam pattern not sensitive to frequency sweeping is in great demand in future wireless applications.

In this paper, we propose a new wideband array antenna system which is composed of two equispaced four-element arrays, phase shifters, diplexers and power dividers. The two arrays are interleaved and connected to diplexers and power dividers. The proposed antenna system associated with feeding network and antenna elements can be integrated in a single substrate, offering lower fabrication and assembly cost and more compact size than the conventional solutions. A wideband antenna array with bandwidth from $2.0 \mathrm{GHz}$ to $6.0 \mathrm{GHz}$ and main beam pointing to 20 degrees is illustrated as an example.

\section{Design and Implementation}

The structure of the proposed system is depicted in Fig. 1. The phase shifters are designed to position the main beam to a specific direction. The use of the diplexers is to switch the two arrays as frequency sweeps. Assume that $f_{1}$ and $f_{2}$ are the lowest and the highest frequencies of the desired band, choose

$$
\begin{gathered}
f_{c}=\sqrt{f_{1} f_{2}} \\
f_{L O}=\frac{\left(f_{1}+f_{c}\right)}{2} \\
f_{H I}=\frac{\left(f_{2}+f_{c}\right)}{2}
\end{gathered}
$$

Such a choice makes the low- and high-bands of the diplexer have the same fractional bandwidth. The frequency response of the diplexer is plotted in Fig. 2. The delay lines in Fig. 1 are designed so that the outputs of the diplexer are with the same phase at $f_{c}$. Finally, the diplexers are fed using wideband power dividers.

The array factor of the proposed system can be expressed in the form

$$
\begin{gathered}
A F(f, \theta)=C_{L O}(f) \sum_{n=1}^{4} e^{j n\left(k_{0} d \sin \theta-\delta_{L O}\right)}+C_{H I}(f) \sum_{n=1}^{4} e^{j n\left(k_{0} s \sin \theta-\delta_{H I}\right)} \\
\delta_{L O, H I}=\delta \frac{f}{f_{L O, H I}}
\end{gathered}
$$

where

$$
\begin{array}{ll}
k_{0} & \text { wave number in free space; } \\
d \text { and } s & \text { half-wavelengths in free space at frequencies } f_{L O} \text { and } f_{H I}, \\
& \text { respectively; }
\end{array}
$$


$C_{L O}$ and $C_{H I}$ frequency responses of the low-band and the high-band of the diplexer, respectively;

$\delta \quad$ system progressive phase factor, a constant;

$\delta_{L O}$ and $\delta_{H I} \quad$ progressive phase factors of array with spacing $d$ and $s$, respectively.

$C_{L O}$ and $C_{H I}$ are similar to the responses of bandpass filters with center frequencies at $f_{L O}$ and $f_{H I}$, respectively. The array with spacing $d$ works as frequency being around $f_{L O}$, and the array with spacing $s$ works as frequency being around $f_{H I}$. By introducing the diplexers, the deformation of the main beam due to frequency sweep can be relieved.

A $2.0 \mathrm{GHz}$ to $6.0 \mathrm{GHz}$ wideband antenna array is illustrated to demonstrate the performance of the proposed system. According to Eqs. (3)-(5), $f_{c}=3.46 \mathrm{GHz}$, $f_{L O}=2.73 \mathrm{GHz}$ and $f_{H I}=4.73 \mathrm{GHz}$. The spacings $d$ and $s$ are $54.9 \mathrm{~mm}$ and 31.7 $\mathrm{mm}$, respectively. The minimal spacing between two antenna elements is 11.6 $\mathrm{mm}$, corresponding to $0.13 \lambda_{0}$ at $f_{c}$. Assume that the mutual couplings between antenna elements can be neglected, the simulated array factor is plotted in Fig. 3 , and the side lobe level against frequency and the $3 \mathrm{~dB}$ beamwidth against frequency are shown in Fig. 4. It is seen that the main beam points to 20 degrees over $2.0 \mathrm{GHz}$ to $6.0 \mathrm{GHz}$, the $3 \mathrm{~dB}$ beamwidth is between $22^{\circ}$ and $38^{\circ}$, and the side lobe level is larger than $10 \mathrm{~dB}$ from $2.0 \mathrm{GHz}$ to $5.7 \mathrm{GHz}$.

The substrate used is an RO4003 with thickness $0.508 \mathrm{~mm}$ and relative dielectric constant 3.38. A diplexer composed of two stub-line filters [3] is designed to meet the curves shown in Fig. 2 whose layout is shown in Fig. 5. Figure 6 presents the simulated and measured results of the designed diplexer. The layout of feeding network is shown in Fig. 7. The antenna element based on monopole antennas is being designed.

\section{Conclusions}

A new wideband planar antenna array has been proposed in this paper. This antenna system associated with feeding network and antenna elements can be integrated in a single substrate. Consequently, such a design is with low fabrication and assembly cost and compact size. So far, the feeding network has been completed for the design example, and the antenna element based on monopole antennas is also being designed. The overall system will be integrated and tested in the near future.

\section{References:}

[1] Robert S. Elliott, Antenna Theory and Design Revised Edition, Wiley-Interscience, 2003, Ch 4.

[2] Tuan Do-Hong and Peter Russer, "Signal Processing for Wideband Smart Antenna Array and Applications," IEEE Microwave Magazine, pp. 57-67, Mar. 2004. 
[3] George L. Matthaei, Leo Young, and E. M. T. Jones, Microwave Filters, Impedance-Matching Networks, and Coupling Structures, McGraw-Hill, 1964, Chapter 16.

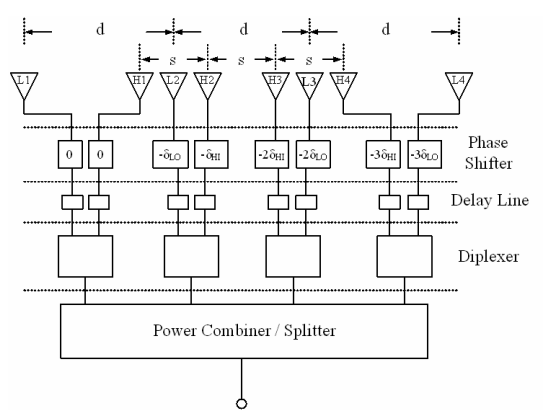

Figure 1. Proposed System Architecture.
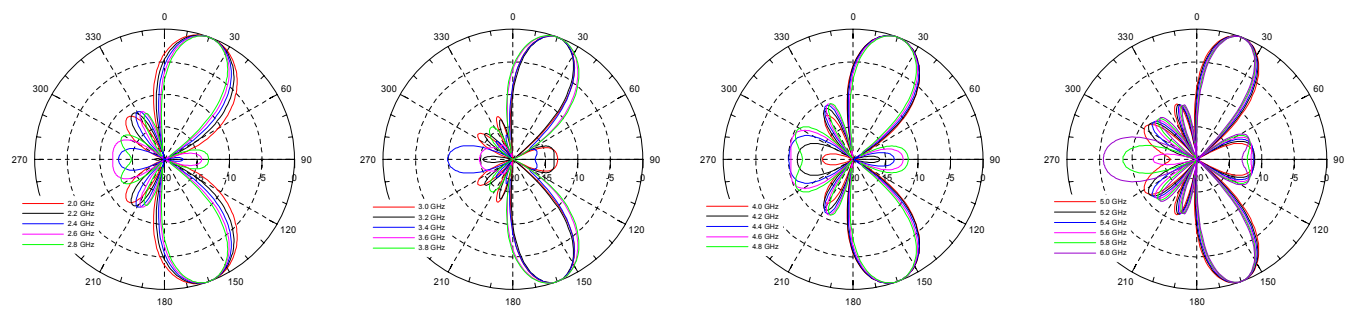

Figure 3. Simulated array factors of the proposed array system.

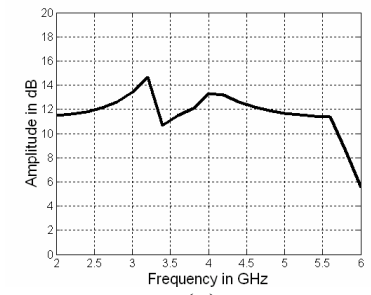

(a)

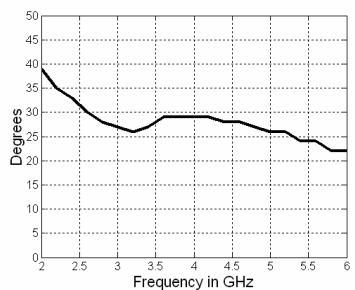

(b)
Figure 4. Side lobe level and $3 \mathrm{~dB}$ beamwidth against frequency. (a) Side lobe level. (b) $3 \mathrm{~dB}$ beamwidth.

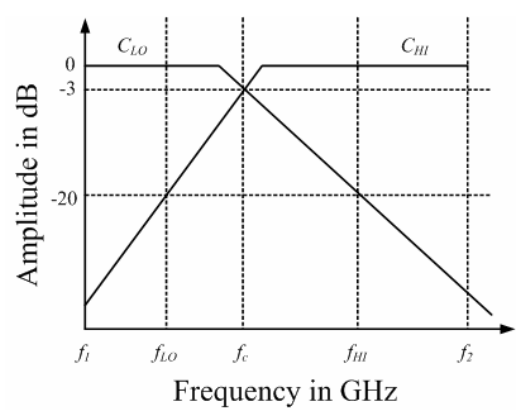

Figure 2. Specification of the diplexer.

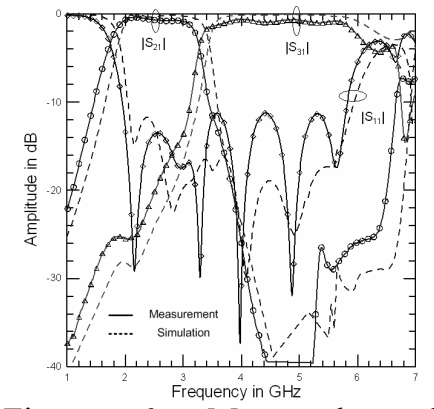

Figure 6. Measured and simulated results of the diplexer shown in Fig. 5.

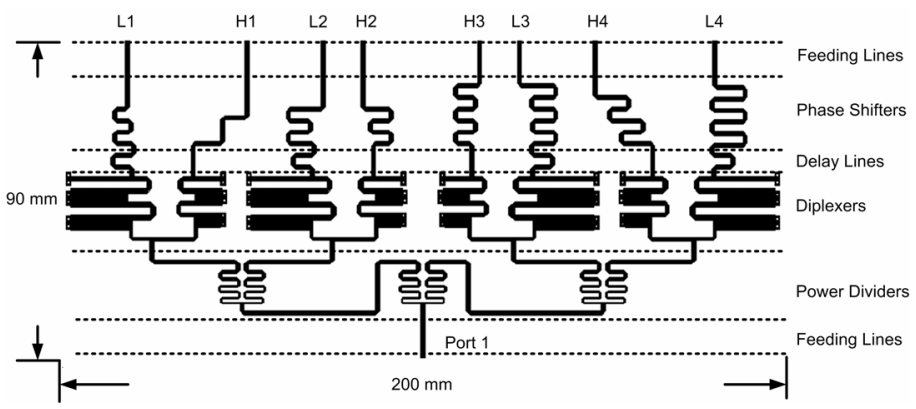

Figure 7. Layout of the feeding network of the designed wideband planar antenna array. 